\title{
Aarco $\mid$ Perfil sociodemográfico e assistencial da morte materna em Recife, 2006-2017: estudo descritivo*
}

\section{Sociodemographic and assistance profile of maternal death in Recife, PE, Brazil, 2006-2017: a descriptive study}

\section{Perfil sociodemográfico y asistencial de la muerte materna en Recife, PE, Brasil, 2006-2017: estudio descriptivo}

\author{
Patrícia Ismael de Carvalho' - D orcid.org/0000-0001-6820-0613 \\ Paulo Germano de Frias ${ }^{1}$ - D orcid.org/0000-0003-4497-8898 \\ Marcelle Luana Carneiro Lemos ${ }^{2}$ - o orcid.org/0000-0002-2167-2602 \\ Luciana Alves Lima de Melo Frutuoso² - o orcid.org/0000-0002-5078-4689 \\ Barbara de Queiroz Figueirôa ${ }^{2}$ - (1) orcid.org/0000-0002-5986-022X \\ Cândida Correia de Barros Pereira² ${ }^{2}$ - Đorcid.org/0000-0001-9456-9721 \\ Idalacy de Carvalho Barreto ${ }^{2}$ - (1) orcid.org/0000-0002-6997-4810 \\ Suely Arruda Vidal' ${ }^{1}$ - (1) orcid.org/0000-0002-4268-520X
}

'Instituto de Medicina Integral Professor Fernando Figueira, Programa de Pós-Graduação em Medicina Integral, Recife, PE, Brasil ${ }^{2}$ Secretaria Estadual de Saúde de Pernambuco, Secretaria Executiva de Vigilância em Saúde, Recife, PE, Brasil

\section{Resumo}

objetivo: descrever características sociodemográficas e assistenciais de mulheres que morreram por causa materna em Recife, Pernambuco, Brasil. Métodos: estudo descritivo utilizando o Sistema de Informações sobre Mortalidade, fichas de investigação e fichas-síntese de óbitos maternos, precoces e tardios, ocorridos entre 2006 e 2017, com evitabilidade avaliada pelo Comitê Municipal de Mortalidade Materna. Resultados: identificaram-se 171 óbitos, 133 no puerpério; a maior parte dos óbitos ocorreu em negras $(68,4 \%)$, sem companheiro $(60,2 \%)$, acompanhadas com atendimento pré-natal $(77,2 \%)$, de parto em maternidades/hospitais $(97,1 \%)$, assistidas por obstetras $(82,6 \%)$; das mulheres com complicações puerperais, 10,4\% não tiveram assistência; óbitos evitáveis/provavelmente evitáveis corresponderam a 81,9\%, por causas indiretas $(n=80)$ e diretas $(n=79)$. Conclusão: as mortes ocorreram principalmente no puerpério e em negras; falhas assistenciais foram frequentes; é necessária melhor vigilância e acompanhamento dos serviços de saúde no período gravídico-puerperal, em Recife.

Palavras-chave: Mortalidade Materna; Complicações na Gravidez; Registros de Mortalidade; Sistemas de Informação em Saúde; Epidemiologia Descritiva.

*Artigo derivado de dados coletados para a tese de doutorado intitulada 'Mortalidade materna em Pernambuco: magnitude, trajetória assistencial e seu custo social', a ser apresentada por Patrícia Ismael de Carvalho junto ao Programa de Pós-Graduação em Medicina Integral/Curso de Doutorado do Instituto de Medicina Integral Professor Fernando Figueira, em 2020. 


\section{Introdução}

A morte materna permanece como um importante problema de saúde em muitas regiões do mundo, principalmente naquelas menos desenvolvidas. ${ }^{1,2} \mathrm{Em} 2015$, a razão de morte materna global estimada foi de 216 por 100 mil nascidos vivos (NV), variando de 542 por 100 mil NV na África a 16 por 100 mil NV na Europa. ${ }^{2}$

\section{A redução da mortalidade materna é uma diretriz mundial, por seu caráter evitável}

Estudo realizado nos Estados Unidos identificou, no período 2011-2015, maiores razões de morte materna entre mulheres negras ( 42,8 por $100 \mathrm{mil} \mathrm{NV})$, sem companheiro (22,8 por 100 mil NV) e com baixa escolaridade. ${ }^{1}$ Condições institucionais também contribuíram para essas mortes, como pouca experiência das equipes de saúde com emergências obstétricas, ausência de pessoal ou serviços especializados, atraso no diagnóstico e falta de continuidade dos cuidados, entre outras. ${ }^{1}$

A redução da mortalidade materna é uma diretriz mundial, por seu caráter evitável. ${ }^{2,3}$ Presente entre os Objetivos de Desenvolvimento do Milênio para 2015, ela foi reiterada nos Objetivos de Desenvolvimento Sustentável para 2030. ${ }^{3} 0$ Brasil não alcançou a meta dos Objetivos do Desenvolvimento do Milênio, ao apresentar uma razão de morte materna de 44 por 100 mil NV em 2015, superior às registradas em países mais pobres da América Latina, como Cuba (39 por 100 mil NV), Costa Rica (25 por 100 mil NV) e Uruguai (15 por 100 mil NV). ${ }^{2}$

0 Nordeste, no triênio 2009-2011, e especialmente Pernambuco, tiveram razões de morte materna de 80,8 por 100 mil NV e 63,3 por 100 mil NV, respectivamente, superiores à média nacional; e possivelmente, ainda maiores, devido ao sub-registro. ${ }^{4}$

No Brasil, o risco de morte materna por causas obstétricas diretas foi de 40,7 por 100 mil NV, aproximadamente o dobro do risco de morte por causas obstétricas indiretas (19,4 por 100 mil NV), em 2012. No mesmo ano, as causas diretas mais frequentes das mortes maternas foram hipertensão arterial $(19,6 \%)$, hemorragia (11,6\%) e infecção puerperal $(7,1 \%)$, ao passo que, entre as causas de morte obstétricas indiretas, destacaram-se as doenças do aparelho circulatório complicadas pela gestação, parto ou puerpério $(7,1 \%)$. Em
Pernambuco, ainda em 2012, a distribuição das causas de morte materna foi semelhante à situação nacional. ${ }^{5}$

Estudos brasileiros prévios revelaram as causas de mortalidade materna e suas características sociodemográficas, sem detalhar os aspectos clínicos, epidemiológicos e assistenciais do ciclo gravídico-puerperal, restringindo-se às variáveis disponíveis nas Declarações de Óbito (D0s) ${ }^{5-8} \mathrm{~A}$ avaliação das informações que fazem parte da investigação epidemiológica dos óbitos maternos no hospital, no ambulatório e no domicílio, regulamentada pelo Ministério da Saúde em 2008, agregariam informações relevantes. ${ }^{9}$ Tais investigações forneceriam subsídios adicionais, contribuindo para explicitar o perfil epidemiológico e sociodemográfico das mulheres vitimadas, esclarecer as circunstâncias do óbito, identificar problemas assistenciais e propor mudanças na atenção à saúde da mulher. ${ }^{1,9}$

Este estudo teve como objetivo descrever características sociodemográficas e assistenciais de mulheres que morreram por causa materna em Recife, Pernambuco, Brasil.

\section{Métodos}

Trata-se de um estudo descritivo realizado a partir dos registros nas D0s, da Ficha Confidencial de Investigação de Óbito Materno e da Ficha de Investigação do Óbito Materno - Síntese. As D0s são preenchidas por médicos da unidade de ocorrência do óbito ou nos serviços de necropsia; as fichas de investigação, em entrevistas familiares, a partir dos prontuários e laudos de necropsia; e a ficha-síntese, na discussão do grupo técnico de mortalidade materna, com base nas múltiplas fontes. ${ }^{10}$

Recife localiza-se no litoral da Zona da Mata pernambucana, possui $218,435 \mathrm{~km}^{2}$ e apresentava, em 2010, uma população de 1.537 .704 habitantes, dos quais 522.120 mulheres em idade fértil ( $10 \mathrm{a} 49$ anos), residentes em 94 bairros exclusivamente urbanos. ${ }^{11} \mathrm{De}$ acordo com o Cadastro Nacional de Estabelecimentos de Saúde (CNES), o município dispõe da maior rede assistencial do estado, com sete maternidades públicas e nove privadas, onde ocorrem, anualmente, $50 \mathrm{mil}$ partos de nascidos vivos, sendo 23 mil residentes no Recife; são 130 unidades básicas de saúde, 276 equipes da Estratégia Saúde da Família (ESF) e 2.100 agentes comunitários de saúde (ACS) para cobrir 51\% da população. ${ }^{12}$ A vigilância do óbito materno foi implantada a partir de 1994. Para os grupos técnicos de discussão, 
são convidados a participar os profissionais da Atenção Primária e dos estabelecimentos da Saúde onde a mulher foi atendida até 0 momento do óbito. ${ }^{13}$

0 estudo avaliou todos os óbitos maternos de residentes no município de Recife, inclusive os classificados como tardios (de 43 dias a um ano após o parto), investigados e discutidos pelo grupo técnico de mortalidade materna da Secretaria de Saúde do Recife, vinculado ao Comitê Municipal de Mortalidade Materna. A inclusão dos óbitos maternos tardios visou ampliar o espectro do estudo, mediante a extensão do prazo de detecção - e inclusão - das mortes maternas postergadas pelo avanço das tecnologias de saúde.

A seguir são descritas as variáveis consideradas para 0 estudo:

a) Sociodemográficas

- idade (em anos: 10-19, 20-34, 35-49);

- raça/cor da pele (branca, negra [incluindo pardas e pretas], ignorada);

- situação conjugal (com companheiro, sem companheiro [inclui solteiras, viúvas ou separadas], ignorada);

- escolaridade (sem escolaridade, ensino fundamental I e II, ensino médio, ensino superior, ignorada); e

- ocupação (dona de casa, trabalhadora do comércio, estudante, empregada doméstica, professora, outras, ignorada).

b) História reprodutiva, gestação e pré-natal

- gravidez anterior (nenhuma, 1, 2 e mais, ignorada);

- tentativa de aborto (sim, não, ignorada);

- vacinação contra tétano (completa, incompleta, não imunizada, ignorada);

- visita do ACS (sim, não, ignorada);

- complicações na gestação (sim, não, ignorada);

- tratamento das complicações na gestação (sim, não, ignorado);

- acompanhamento pré-natal (sim, não, ignorado);

- número de consultas no pré-natal (1-3, 4-6, 7 e mais, ignorado);

- tipo de estabelecimento de saúde do pré-natal (unidade de Saúde da Família, hospital público/filantrópico/ policlínica, hospital/consultório particular, ignorado);

- idade gestacional na primeira consulta de pré-natal (em semanas: 1-13, 14-26, 27-42, ignorada);

- cartão de pré-natal, ou Cartão da Gestante (sim, não, ignorado);

- pressão arterial na primeira consulta de pré-natal (normal, elevada, ignorada);

- encaminhamento ao pré-natal de alto risco (sim, não, ignorado);

- consulta no pré-natal de alto risco (sim, não, ignorada);

- tipo de estabelecimento do pré-natal de alto risco (hospital público/filantrópico/policlínica, consultório particular, ignorado).

c) Parto e aborto (idade gestacional $<20$ semanas)

- local do parto/aborto (maternidade, hospital geral, domicílio, Serviço de Atendimento Móvel de Urgência [SAMU], ignorado);

- tipo de estabelecimento do parto/aborto (hospital privado, hospital público/filantrópico/policlínica/SAMU, ignorado);

- município do estabelecimento onde ocorreu o parto/ aborto (Recife, outros);

- idade gestacional no parto/aborto (em semanas: $\leq 22,23-36,37-41, \geq 42$, ignorada);

- transferência durante 0 parto/aborto (sim, não, sem internação);

- tipo de aborto (espontâneo, induzido pela mulher, retido);

- procedimentos usados no aborto induzido (chá caseiro, misoprostol, outros);

- apresentou complicações decorrentes do aborto (sim, não, ignorado);

- complicações decorrentes do aborto (infecção, hemorragia, embolia, perfuração uterina, outras);

- tipo de parto (vaginal, cesáreo, vaginal e cesáreo [quando ocorrem os dois tipos, na mesma gestação]);

- duração do trabalho de parto (em horas: $\leq 3,4-12$, $>12$, ignorada);

- profissional que realizou o parto (obstetra, outro, ignorado);

- anamnese preenchida (sim, não, ignorada);

- partograma preenchido (sim, não, ignorado);

- acompanhante no trabalho de parto (sim, não, ignorado);

- apresentou complicações no parto (sim, não, ignorado); e

- complicações no parto (hemorragia; sofrimento fetal; pré-eclâmpsia; feto morto; eclâmpsia; síndrome de hemolysis, elevated liver enzymes and low platelets [HELLP]; outras).

d) Puerpério e circunstâncias do óbito

- apresentou complicações no puerpério (sim, não, ignorado);

- tratamento das complicações no puerpério (sim, não, ignorado); 
- complicações no puerpério (infecções, hemorragia, problemas cardiovasculares, eclâmpsia, embolia, neoplasias, atonia uterina, outras);

- momento do óbito (durante a gestação, no trabalho de parto, puerpério precoce, puerpério tardio);

- local do óbito (hospital, outros estabelecimentos de saúde, domicílio);

- município de ocorrência do óbito (Recife, outros);

- critério de evitabilidade do óbito, preconizado no Guia de Vigilância Epidemiológica do Óbito Materno, o qual não se limita à análise das medidas terapêuticas mas considera fatores relacionados à comunidade e à mulher, profissionais, institucionais, sociais e intersetoriais, e que após análise singular de cada caso pelo Comitê de Mortalidade Materna, classifica o óbito (evitável, provavelmente evitável, provavelmente inevitável, inconclusivo, ignorado) $;{ }^{10}$

- realização de necropsia (sim, não, ignorada);

- análise de útero e anexos na necropsia (sim, não, ignorada);

- causas obstétricas diretas, definidas como resultantes de complicações da gravidez, parto ou puerpério, devidas a intervenções, omissões, tratamento incorreto, ou à cadeia de eventos decorrente de qualquer uma dessas causas ${ }^{10}$ (hipertensão, embolia, hemorragia, infecção puerperal, aborto, gravidez que termina em aborto, outras complicações relacionadas ao trabalho de parto e parto, infecções do trato geniturinário na gravidez, cardiomiopatia no puerpério, demais causas);

- causas obstétricas indiretas, resultantes de doença prévia da mãe ou desenvolvida durante a gravidez, não devida a causas obstétricas diretas mas agravada pelos efeitos fisiológicos da gravidez ${ }^{10}$ (doenças do aparelho circulatório, doenças e afecções especificadas, doenças do aparelho respiratório, HIV/aids, hipertensão preexistente, doenças infectoparasitárias complicando a gravidez, o parto e o puerpéreo, transtornos mentais, doenças do aparelho digestivo, outras doenças do sangue, doenças endócrinas);

- morte obstétrica de causa não especificada;

- morte por qualquer causa obstétrica tardia (43 dias a 1 ano); e

- causa mal definida.

As causas de óbito consideradas estão catalogadas no capítulo XVIII da Classificação Estatística Internacional de Doenças e Problemas Relacionados à Saúde - Décima Revisão (CID-10). 0s dados do estudo foram levantados do Sistema de Informações sobre Mortalidade (SIM), da Ficha Confidencial de Investigação de Óbito Materno e da Ficha de Investigação do Óbito Materno - Síntese, ${ }^{10}$ referentes aos óbitos ocorridos no período de $1^{\circ}$ de janeiro de 2006 a 31 de dezembro de 2017, cedidos pela Secretaria Estadual de Saúde de Pernambuco e Secretaria de Saúde do Recife, consultados e coletados no segundo semestre de 2018.

0s dados provindos do SIM foram tabulados no TabWin32, e os da ficha-síntese, extraídos do banco da vigilância do óbito materno, ambos conjuntos de dados digitados pelo programa Epi Info versão 3.6.3, sendo utilizados os módulos make view e enter data. As variáveis da Ficha Confidencial de Investigação de Óbito Materno geraram um banco de dados em planilha eletrônica Excel® 2007. A consolidação em um banco único de dados foi possível pela técnica de linkage determinístico, na plataforma Excel® 2007, utilizando-se como variável-chave o número da declaração de óbito. Todas as D0s e fichas de investigação do óbito materno com problemas no preenchimento foram complementadas pela busca de informações em diversas fontes (hospital, domicílio e ambulatório), realizada por técnicos da Secretaria de Saúde do Recife.

Incluiu-se o universo de mulheres idas a óbito por causas maternas precoces e tardias notificadas, a que se somaram as identificadas após a investigação dos óbitos daquelas em idade fértil, dentro do período estudado.

Realizaram-se estatísticas descritivas, apresentadas em distribuição de frequência absoluta e relativa para o total dos anos do período. Foram calculadas proporções dos óbitos maternos segundo as variáveis do estudo.

0 projeto do estudo foi aprovado pelo Comitê de Ética em Pesquisa do Instituto de Medicina Integral Professor Fernando Figueira: Parecer n ${ }^{0}$ 2.277.245, de 15/9/2017; Certificado de Apresentação para Apreciação Ética (CAAE) n n 72815317.4.0000.5201.

\section{Resultados}

Dos 6.921 óbitos de mulheres em idade fértil residentes no Recife, $76,8 \%$ foram investigados, e destes, $171(3,2 \%)$ classificados como morte materna após discussão técnica, 35 ocorridos durante a gravidez, três no trabalho de parto e 133 no puerpério (Figura 1). 


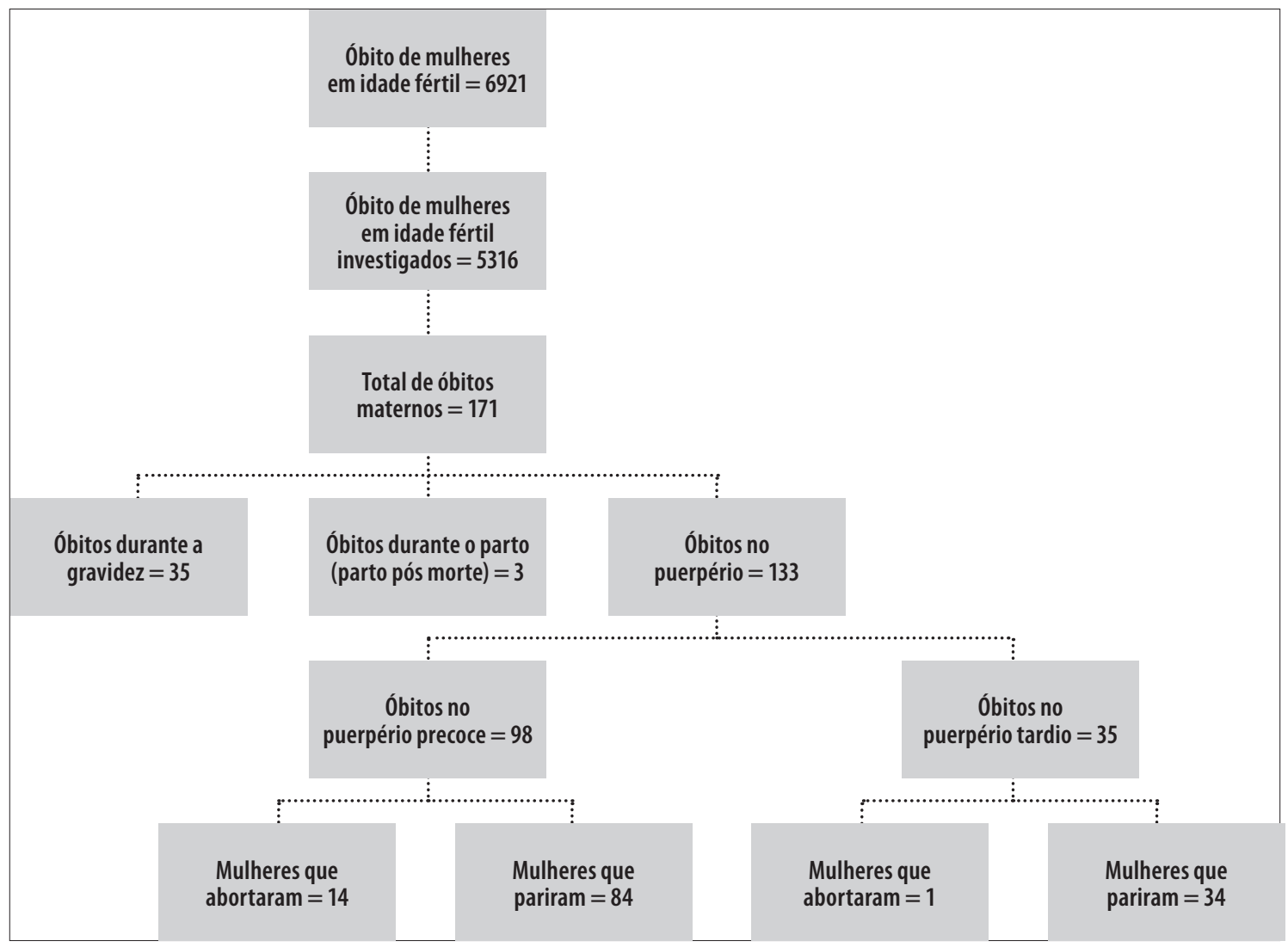

Figura 1 - Óbitos maternos segundo momento de ocorrência, Recife, Pernambuco, 2006-2017

Entre as mulheres falecidas, $84,2 \%(\mathrm{n}=144)$ se encontravam na idade de 20 a 39 anos, $68,4 \%(n=117)$ eram negras, $60,2 \%(n=103)$ viviam sem companheiro, 34,5\% ( $\mathrm{n}=59)$ estudaram até 0 ensino fundamental ou ensino médio e 46,2\% $(n=79)$ eram donas de casa. Não foram registrados óbitos maternos em mulheres indígenas e amarelas (Tabela 1).

Dessas mulheres, $35(20,5 \%)$ não realizaram 0 acompanhamento pré-natal, e destas, 19 faleceram no puerpério. Entre as que fizeram pré-natal, 35 (26,5\%) compareceram a até três consultas; destas, cinco morreram durante a gravidez. A maioria das consultas de pré-natal $(59,1 \% ; n=78)$ ocorreu em unidades da $\mathrm{ESF}$, $34,8 \%(n=46)$ iniciaram o acompanhamento durante 0 primeiro trimestre da gravidez, $64,4 \%(\mathrm{n}=85)$ receberam o cartão de pré-natal (Cartão da Gestante) e $7,6 \%(\mathrm{n}=10)$ apresentaram pressão arterial elevada na primeira consulta. Complicações na gestação ocorreram em 73,1\% (n=125) das mulheres e 46,4\% (n=58) receberam tratamento em razão dessas complicações; $42,4 \%(n=56)$ das mulheres foram encaminhadas ao pré-natal de alto risco, e destas, $85,7 \%(\mathrm{n}=48)$ realizaram consultas, das quais $87,4 \%(n=42)$ em hospital público/filantrópico/policlínica. Encontrou-se 67,8\% $(\mathrm{n}=116)$ de informação ignorada sobre imunização contra o tétano e 54,4\% ( $n=93)$ de informação ignorada sobre tentativa de aborto na gestação atual (Tabela 2).

0 principal local da assistência ao parto e ao aborto, para $92,7 \%(n=126)$ das mulheres, foram as maternidades, das quais $90,2 \%(\mathrm{n}=120)$ localizadas no Recife; $19,5 \%(n=26)$ desses procedimentos ocorreram em estabelecimentos de saúde de caráter privado. Dos 171 óbitos maternos estudados, 121 mulheres pariram, e destas, 105 tiveram filhos nascidos vivos. Entre os 15 abortos registrados, nove foram espontâneos e quatro induzidos pela mulher. Em dez dos abortos, ocorreram complicações, seis por infecção e quatro por hemorragias. A idade gestacional no momento do parto, para 46,3\% $(n=63)$ das mulheres, foi entre 23 e 36 semanas. A maioria dos partos foi realizada por médicos obstetras $(82,6 \% ; n=100), 61,2 \%(n=74)$ desses partos cirúrgicos (Tabela 3 ). 
Tabela 1 - Frequência das características sociodemográficas de mulheres que morreram por causas maternas ( $n=171$ ), Recife, Pernambuco, 2006-2017

\begin{tabular}{|c|c|c|}
\hline Características & $\mathbf{n}$ & $\%$ \\
\hline \multicolumn{3}{|l|}{ Idade (em anos) } \\
\hline $10-19$ & 14 & 8,2 \\
\hline $20-34$ & 118 & 69,0 \\
\hline $35-49$ & 39 & 22,8 \\
\hline \multicolumn{3}{|l|}{ Raça/cor da pele } \\
\hline Branca & 51 & 29,8 \\
\hline Negra & 117 & 68,4 \\
\hline Ignorada ${ }^{a}$ & 3 & 1,8 \\
\hline \multicolumn{3}{|l|}{ Situação conjugal } \\
\hline Com companheiro & 65 & 38,0 \\
\hline Sem companheiro & 103 & 60,2 \\
\hline Ignorada ${ }^{a}$ & 3 & 1,8 \\
\hline \multicolumn{3}{|l|}{ Escolaridade } \\
\hline Sem escolaridade & 15 & 8,8 \\
\hline Ensino fundamental I e II & 59 & 34,5 \\
\hline Ensino médio & 59 & 34,5 \\
\hline Ensino superior & 20 & 11,7 \\
\hline Ignorada ${ }^{a}$ & 18 & 10,5 \\
\hline \multicolumn{3}{|l|}{ Ocupação } \\
\hline Dona de casa & 79 & 46,2 \\
\hline Trabalhadora do comércio & 20 & 11,7 \\
\hline Estudante & 16 & 9,4 \\
\hline Empregada doméstica & 16 & 9,4 \\
\hline Professora & 6 & 3,5 \\
\hline Outras & 30 & 17,5 \\
\hline $\operatorname{lgnorado} 0^{\mathbf{a}}$ & 4 & 2,3 \\
\hline
\end{tabular}

a) Corresponde às alternativas 'Ignorado' e 'Em branco', decorrentes das repostas disponíveis nos instrumentos utilizados.

Não havia registro da anamnese em 28 dos prontuários $(23,1 \%)$, como também não havia registro do partograma em 72 (59,5\%). Verificou-se $38,0 \%(n=46)$ de complicações do parto, sendo hemorragia (30,4\%; $\mathrm{n}=14)$ e sofrimento fetal $(21,7 \% ; \mathrm{n}=10)$ as principais. Em 18,2\% ( $\mathrm{n}=22)$ dos partos, a mulher não contou com a presença do acompanhante (Tabela 3).

As mortes maternas ocorreram, predominantemente, no puerpério precoce $(57,3 \% ; \mathrm{n}=98)$, durante a gestação e no puerpério tardio (para cada um: $20,5 \% ; n=35$ ), e no trabalho de parto $(1,7 \% ; n=3)$. Em $94 \%(n=125)$ dessas mortes, ocorreram complicações no puerpério, devidas sobretudo a infecções $(24,0 \% ; n=30)$, hemorragias $(23,2 \% ; n=29)$ e problemas cardiovasculares $(19,2 \%$; $\mathrm{n}=24)$. Dos casos de complicação no puerpério, $10,4 \%$ $(\mathrm{n}=13)$ não tiveram tratamento. Quase metade $(47,4 \%$; $\mathrm{n}=81$ ) dos corpos foram necropsiados, e destes, $69,2 \%$ $(\mathrm{n}=56)$ tiveram o útero e anexos examinados (Tabela 4).

Foram classificados como evitáveis e provavelmente evitáveis $81,9 \%(\mathrm{n}=140)$ dos óbitos maternos, dos quais as causas obstétricas indiretas representaram $46,8 \%$ $(n=80)$, e as diretas, $46,2 \%(n=79)$. Entre as causas obs- 
Tabela 2 - Frequência das características reprodutivas, gestação e pré-natal de mulheres morreram por causas maternas ( $n=171)$, Recife, Pernambuco, 2006-2017

\begin{tabular}{|c|c|c|}
\hline Características & n & $\%$ \\
\hline \multicolumn{3}{|c|}{ Gravidez anterior } \\
\hline Nenhuma & 49 & 28,7 \\
\hline 1 & 43 & 25,1 \\
\hline 2 e mais & 74 & 43,3 \\
\hline Ignorada ${ }^{a}$ & 5 & 2,9 \\
\hline \multicolumn{3}{|c|}{ Tentativa de aborto } \\
\hline Sim & 5 & 2,9 \\
\hline Não & 73 & 42,7 \\
\hline Ignorada ${ }^{a}$ & 93 & 54,4 \\
\hline \multicolumn{3}{|c|}{ Vacinação contra tétano } \\
\hline Completa & 40 & 23,4 \\
\hline Incompleta & 13 & 7,6 \\
\hline Não imunizada & 2 & 1,2 \\
\hline Ignorada ${ }^{a}$ & 116 & 67,8 \\
\hline \multicolumn{3}{|c|}{ Visita do agente comunitário de saúde } \\
\hline Sim & 57 & 33,3 \\
\hline Não & 45 & 26,3 \\
\hline Ignorada ${ }^{a}$ & 69 & 40,4 \\
\hline \multicolumn{3}{|c|}{ Complicações na gestação } \\
\hline Sim & 125 & 73,1 \\
\hline Não & 21 & 12,3 \\
\hline Ignorado ${ }^{a}$ & 25 & 14,6 \\
\hline \multicolumn{3}{|c|}{ Tratamento das complicações na gestaçãob } \\
\hline Sim & 58 & 46,4 \\
\hline Não & 35 & 28,0 \\
\hline $\operatorname{lgnorado} a^{a}$ & 32 & 25,6 \\
\hline \multicolumn{3}{|c|}{ Acompanhamento pré-natal } \\
\hline Sim & 132 & 77,2 \\
\hline Não & 35 & 20,5 \\
\hline $\operatorname{lgnorado} 0^{a}$ & 4 & 2,3 \\
\hline \multicolumn{3}{|c|}{ Número de consultas no pré-natalc } \\
\hline $1-3$ & 35 & 26,5 \\
\hline $4-6$ & 40 & 30,3 \\
\hline 7 e mais & 39 & 29,6 \\
\hline Ignorado ${ }^{a}$ & 18 & 13,6 \\
\hline
\end{tabular}


Tabela 2 - Frequência das características reprodutivas, gestação e pré-natal de mulheres morreram por causas maternas ( $n=171$ ), Recife, Pernambuco, 2006-2017

\begin{tabular}{|c|c|c|}
\hline Características & $\mathbf{n}$ & $\%$ \\
\hline \multicolumn{3}{|c|}{ Tipo de estabelecimento de saúde do pré-natal' } \\
\hline Unidade da Estratégia Saúde da Família (ESF) & 78 & 59,1 \\
\hline Hospital público/filantrópico/policlínica & 27 & 20,4 \\
\hline Hospital/consultório particular & 24 & 18,2 \\
\hline Ignorado ${ }^{a}$ & 3 & 2,3 \\
\hline \multicolumn{3}{|c|}{ Idade gestacional na primeira consulta de pré-natal (em semanas)` } \\
\hline $1-13$ & 46 & 34,8 \\
\hline $14-26$ & 45 & 34,1 \\
\hline $27-42$ & 3 & 2,3 \\
\hline Ignorada ${ }^{a}$ & 38 & 28,8 \\
\hline \multicolumn{3}{|l|}{ Cartão de pré-natal (Cartão da Gestante)c } \\
\hline Sim & 85 & 64,4 \\
\hline Não & 7 & 5,3 \\
\hline Ignorado ${ }^{a}$ & 40 & 30,3 \\
\hline \multicolumn{3}{|c|}{ Pressão arterial na primeira consulta de pré-natal' } \\
\hline Normal & 84 & 63,6 \\
\hline Elevada & 10 & 7,6 \\
\hline Ignorada ${ }^{a}$ & 38 & 28,8 \\
\hline \multicolumn{3}{|l|}{ Encaminhamento ao pré-natal de alto riscoc } \\
\hline Sim & 56 & 42,4 \\
\hline Não & 54 & 40,9 \\
\hline Ignorado ${ }^{a}$ & 22 & 16,7 \\
\hline \multicolumn{3}{|l|}{ Consulta no pré-natal de alto risco ${ }^{\mathrm{d}}$} \\
\hline Sim & 48 & 85,7 \\
\hline Não & 6 & 10,7 \\
\hline Ignorada ${ }^{a}$ & 2 & 3,6 \\
\hline \multicolumn{3}{|c|}{ Tipo de estabelecimento do pré-natal de alto riscoe } \\
\hline Hospital público/filantrópico/policlínica & 42 & 87,4 \\
\hline Consultório particular & 3 & 6,3 \\
\hline Ignorado ${ }^{\mathrm{a}}$ & 3 & 6,3 \\
\hline
\end{tabular}

a) Corresponde às alternativas 'Ignorado' $\mathrm{e}^{\prime} \mathrm{Em}$ branco', decorrentes das repostas disponiveis nos instrumentos utilizados.

b) $n=125$ mulheres falecidas que tiveram complicações na gestação.

c) $n=132$ mulheres que realizaram consultas de pré-natal.

d) $n=56$ mulheres que foram encaminhadas ao pré-natal de alto risco.

e) $n=48$ mulheres que realizaram consulta no pré-natal de alto risco. 
Tabela 3 - Frequência das características do parto e aborto de mulheres que morreram por causas maternas ( $n=171)$, Recife, Pernambuco, 2006-2017

\begin{tabular}{|c|c|c|}
\hline Características & $\mathbf{n}$ & $\%$ \\
\hline \multicolumn{3}{|l|}{ Local do parto/aborto ${ }^{\mathrm{a}}$} \\
\hline Maternidade & 126 & 92,7 \\
\hline Hospital geral & 6 & 4,4 \\
\hline Domicílio & 2 & 1,5 \\
\hline Serviço de Atendimento Móvel de Urgência (SAMU) & 1 & 0,7 \\
\hline Ignoradob & 1 & 0,7 \\
\hline \multicolumn{3}{|l|}{ Tipo de estabelecimento do parto/abortoc } \\
\hline Hospital privado & 26 & 19,5 \\
\hline Hospital público/filantrópico/policlínica/SAMU & 104 & 78,2 \\
\hline Ignorado ${ }^{b}$ & 3 & 2,3 \\
\hline \multicolumn{3}{|l|}{ Município do estabelecimento onde ocorreu o parto/abortoc } \\
\hline Recife & 120 & 90,2 \\
\hline Outros & 13 & 9,8 \\
\hline \multicolumn{3}{|l|}{ Idade gestacional no parto/aborto (em semanas) ${ }^{\mathrm{a}}$} \\
\hline$\leq 22$ & 11 & 8,1 \\
\hline $23-36$ & 63 & 46,3 \\
\hline $37-41$ & 42 & 30,9 \\
\hline$\geq 42$ & 2 & 1,5 \\
\hline Ignorada ${ }^{\mathbf{b}}$ & 18 & 13,2 \\
\hline \multicolumn{3}{|l|}{ Transferência durante o parto/aborto ${ }^{\mathrm{a}}$} \\
\hline Sim & 21 & 15,4 \\
\hline Não & 113 & 83,1 \\
\hline Sem internação & 2 & 1,5 \\
\hline \multicolumn{3}{|l|}{ Tipo de aborto ${ }^{d}$} \\
\hline Espontâneo & 9 & 60,0 \\
\hline Induzido pela mulher & 4 & 26,7 \\
\hline Retido & 2 & 13,3 \\
\hline \multicolumn{3}{|l|}{ Procedimentos usados no aborto induzido ${ }^{e}$} \\
\hline Chá caseiro & 1 & 25,0 \\
\hline Misoprostol & 1 & 25,0 \\
\hline Outros & 2 & 50,0 \\
\hline 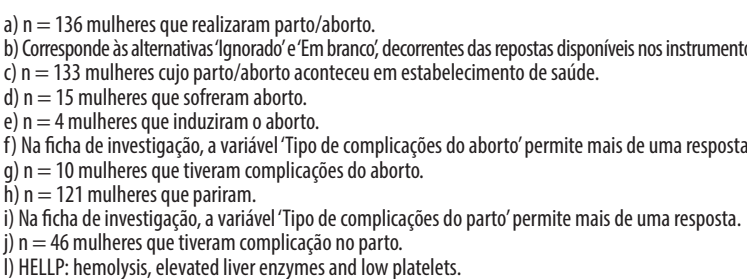 & & \\
\hline
\end{tabular}


Tabela 3 - Frequência das características do parto e aborto de mulheres que morreram por causas maternas ( $n=171$ ), Recife, Pernambuco, 2006-2017

\begin{tabular}{|c|c|c|}
\hline Características & n & $\%$ \\
\hline \multicolumn{3}{|c|}{ Apresentou complicações decorrentes do aborto ${ }^{d}$} \\
\hline Sim & 10 & 66,7 \\
\hline Não & 3 & 20,0 \\
\hline Ignorado $\mathbf{b}$ & 2 & 13,3 \\
\hline \multicolumn{3}{|l|}{ Complicações ${ }^{f}$ decorrentes do aborto ${ }^{g}$} \\
\hline Infecção & 6 & 60,0 \\
\hline Hemorragia & 4 & 40,0 \\
\hline Embolia & 1 & 10,0 \\
\hline Perfuração uterina & 1 & 10,0 \\
\hline Outras & 4 & 40,0 \\
\hline \multicolumn{3}{|l|}{ Tipo de partob } \\
\hline Vaginal & 45 & 37,2 \\
\hline Cesáreo & 74 & 61,2 \\
\hline Vaginal e cesáreo (na mesma gestação) & 2 & 1,6 \\
\hline \multicolumn{3}{|c|}{ Duração do trabalho de parto (em horash) } \\
\hline$\leq 3$ & 10 & 8,3 \\
\hline $4-12$ & 12 & 9,9 \\
\hline$>12$ & 8 & 6,6 \\
\hline Ignoradab & 91 & 75,2 \\
\hline \multicolumn{3}{|l|}{ Profissional que realizou o parto ${ }^{\mathrm{h}}$} \\
\hline Obstetra & 100 & 82,6 \\
\hline Outros & - & - \\
\hline Ignorado $0^{b}$ & 21 & 17,4 \\
\hline \multicolumn{3}{|l|}{ Anamnese preenchidah } \\
\hline Sim & 45 & 37,2 \\
\hline Não & 28 & 23,1 \\
\hline Ignorado $0^{b}$ & 48 & 39,7 \\
\hline \multicolumn{3}{|l|}{ Partograma preenchido ${ }^{h}$} \\
\hline Sim & 9 & 7,4 \\
\hline Não & 72 & 59,5 \\
\hline Ignorado ${ }^{b}$ & 40 & 33,1 \\
\hline \multicolumn{3}{|l|}{ Acompanhante no trabalho de parto ${ }^{h}$} \\
\hline Sim & 30 & 24,8 \\
\hline
\end{tabular}

a) $n=136$ mulheres que realizaram parto/aborto.

b) Corresponde às alternativas'Ignorado' $\mathrm{e}^{\prime} \mathrm{Em}$ branco', decorrentes das repostas disponíveis nos instrumentos utilizados.

c) $n=133$ mulheres cujo parto/aborto aconteceu em estabelecimento de saúde.

d) $n=15$ mulheres que sofreram aborto.

e) $n=4$ mulheres que induziram 0 aborto.

f) Na ficha de investigação, a variável 'Tipo de complicações do aborto' permite mais de uma resposta.

g) $n=10$ mulheres que tiveram complicações do aborto.

h) $n=121$ mulheres que pariram.

i) Na ficha de investigação, a variável'Tipo de complicações do parto' permite mais de uma resposta.

j) $n=46$ mulheres que tiveram complicação no parto.

I) HELLP: hemolysis, elevated liver enzymes and low platelets. 
Tabela 3 - Frequência das características do parto e aborto de mulheres que morreram por causas maternas ( $n=171)$, Recife, Pernambuco, 2006-2017

\begin{tabular}{|c|c|c|}
\hline Características & n & $\%$ \\
\hline Não & 22 & 18,2 \\
\hline Ignorado ${ }^{b}$ & 69 & 57,0 \\
\hline \multicolumn{3}{|c|}{ Apresentou complicações no parto ${ }^{\mathrm{h}}$} \\
\hline Sim & 46 & 38,0 \\
\hline Não & 58 & 47,9 \\
\hline Ignorado ${ }^{b}$ & 17 & 14,1 \\
\hline \multicolumn{3}{|c|}{ Complicações' no parto ${ }^{j}$} \\
\hline Hemorragia & 14 & 30,4 \\
\hline Sofrimento fetal & 10 & 21,7 \\
\hline Pré-eclâmpsia & 9 & 19,6 \\
\hline Feto morto & 6 & 13,0 \\
\hline Eclâmpsia & 5 & 10,9 \\
\hline Síndrome HELLPI & 3 & 6,5 \\
\hline Outras & 47 & 102,2 \\
\hline
\end{tabular}

a) $n=136$ mulheres que realizaram parto/aborto.

b) Corresponde às alternativas'Ignorado' $e^{\prime} E m$ branco,' decorrentes das repostas disponíveis nos instrumentos utilizados.

c) $n=133$ mulheres cujo parto/aborto aconteceu em estabelecimento de saúde.

d) $n=15$ mulheres que sofreram aborto.
e) $n=4$ mulheres que induziram 0 aborto.

f) Na ficha de investigação, a variável 'Tipo de complicações do aborto' permite mais de uma resposta.

g) $n=10$ mulheres que tiveram complicações do aborto.

h) $n=121$ mulheres que pariram.

i) Na ficha de investigação, a variável 'Tipo de complicações do parto' permite mais de uma resposta.

j) $n=46$ mulheres que tiveram complicação no parto.

I) HELLP: hemolysis, elevated liver enzymes and low platelets.

tétricas indiretas, as doenças do aparelho circulatório complicando a gravidez, o parto e o puerpério (CID-10: 099.4) ocasionaram o óbito de 21 mulheres (12,4\%). Entre as diretas, a hipertensão arterial (CID-10: 011/ 014/015) foi responsável por 17 dos óbitos maternos consultados (9,9\%) (Tabela 4).

\section{Discussão}

As mortes maternas foram mais frequentes entre mulheres negras, sem emprego formal, sem companheiro, com início tardio do acompanhamento pré-natal, e realização de quatro a seis consultas de pré-natal. Entre as mulheres que tiveram complicações na gravidez, nem todas foram atendidas no pré-natal de alto risco, e das que apresentaram complicações no puerpério, uma parcela não foi tratada. Os partos/abortos ocorreram, principalmente, em maternidades públicas ou filantrópicas, realizados por médicos obstetras. As principais complicações do aborto foram infecções e hemorragias; no puerpério, além destas, foram registrados problemas circulatórios e respiratórios. A maioria dos óbitos maternos era evitável, destacando-se, entre as causas obstétricas diretas, a hipertensão arterial, embolia e hemorragia; entre as indiretas, prevaleceram as doenças do aparelho circulatório complicando a gravidez, o parto e o puerpério.

A utilização de dados secundários representa limitações: (i) possibilidade de sub-registro da causa do óbito materno na declaração de óbito, (ii) incompletude no preenchimento da D0 e das fichas de investigação, além de (iii) problemas na confiabilidade das informações da D0 e da Ficha Confidencial de Investigação de Óbito Materno, limitando a validade dos resultados apresentados. Essas limitações foram minoradas pela ação da Secretaria de Saúde do Recife no resgate de variáveis nos estabelecimentos de saúde, e pela discussão dos casos no grupo técnico do Comitê Municipal de Mortalidade Materna do Recife. A despeito dos problemas 
de qualidade das informações, trata-se das únicas fontes de dados disponíveis para o monitoramento da mortalidade materna.

0 perfil socioeconômico observado é similar ao do país, sobretudo nas características que vulnerabilizam a mulher, como pobreza e raça/cor da pele..$^{13-15} 0$ recorte étnico-racial expõe as desigualdades da sociedade, a condição socioeconômica e a iniquidade no acesso aos serviços de saúde dirigidos a essa população. ${ }^{15}$ Mulheres negras têm ainda maior probabilidade de viver em contextos desfavoráveis, nos quais as fragilidades sócio-históricas são potencializadas pela violência de gênero, estigma, pobreza e violação dos direitos humanos. ${ }^{14}$ Maior taxa de hipertensão arterial, diabetes mellitus, tabagismo e sífilis trazem, como consequência, desfechos de morte materna mais prevalentes nesse grupo feminino específico. ${ }^{14,15}$

Estudo transversal com mulheres em idade fértil realizado em capitais brasileiras, no primeiro semestre de 2002, identificou maior risco de morte materna entre aquelas com menos de quatro anos de estudo. ${ }^{16}$ Pesquisas posteriores sobre óbitos maternos mostram

\section{Tabela 4 - Frequência das características do puerpério e das circunstâncias do óbito de mulheres que morreram por causas maternas ( $\mathrm{n}=171$ ), Recife, Pernambuco, 2006-2017}

\begin{tabular}{|c|c|c|}
\hline Características & $\mathbf{n}$ & $\%$ \\
\hline \multicolumn{3}{|c|}{ Apresentou complicações no puerpério ${ }^{a}$} \\
\hline Sim & 125 & 94,0 \\
\hline Não & 2 & 1,5 \\
\hline Ignorado & 6 & 4,5 \\
\hline \multicolumn{3}{|c|}{ Tratamento das complicações no puerpérioc } \\
\hline Sim & 108 & 86,4 \\
\hline Não & 13 & 10,4 \\
\hline Ignorado ${ }^{b}$ & 4 & 3,2 \\
\hline \multicolumn{3}{|l|}{ Complicações ${ }^{d}$ no puerpério } \\
\hline Infecções & 30 & 24,0 \\
\hline Hemorragia & 29 & 23,2 \\
\hline Problemas cardiovasculares & 24 & 19,2 \\
\hline Problemas respiratórios & 18 & 14,4 \\
\hline Eclâmpsia & 16 & 12,8 \\
\hline Embolia & 9 & 7,2 \\
\hline Neoplasias & 8 & 6,4 \\
\hline Atonia uterina & 6 & 4,8 \\
\hline Outras & 27 & 21,6 \\
\hline \multicolumn{3}{|l|}{ Momento do óbito } \\
\hline Durante a gestação & 35 & 20,5 \\
\hline No trabalho de parto & 3 & 1,7 \\
\hline Puerpério precoce & 98 & 57,3 \\
\hline Puerpério tardio & 35 & 20,5 \\
\hline
\end{tabular}

a) $n=133$ mulheres que morreram no puerpério.

b) Corresponde às alternativas'Ignorado' $e^{\prime} E \mathrm{Em}$ branco', decorrentes das repostas disponíveis nos instrumentos utilizados.

c) $n=125$ mulheres que sofreram complicaçōes no puerpério.

d) Na ficha de investigação, a variável 'Tipo de complicaçōes do aborto' permite mais de uma resposta.

e) $n=81$ mulheres que passaram por necropsia. 
Tabela 4 - Frequência das características do puerpério e das circunstâncias do óbito de mulheres que morreram por causas maternas ( $n=171$ ), Recife, Pernambuco, 2006-2017

\begin{tabular}{|c|c|c|}
\hline Características & $\mathbf{n}$ & $\%$ \\
\hline \multicolumn{3}{|l|}{ Local do óbito } \\
\hline Hospital & 163 & 95,3 \\
\hline Outros estabelecimentos de saúde & 2 & 1,2 \\
\hline Domicílio & 6 & 3,5 \\
\hline \multicolumn{3}{|l|}{ Município de ocorrência do óbito } \\
\hline Recife & 160 & 93,6 \\
\hline Outros & 11 & 6,4 \\
\hline \multicolumn{3}{|l|}{ Critério de evitabilidade do óbito } \\
\hline Evitável & 80 & 46,8 \\
\hline Provavelmente evitável & 60 & 35,1 \\
\hline Provavelmente inevitável & 24 & 14,0 \\
\hline Inconclusivo & 5 & 2,9 \\
\hline Ignorado ${ }^{b}$ & 2 & 1,2 \\
\hline \multicolumn{3}{|l|}{ Realização de necropsia } \\
\hline Sim & 81 & 47,4 \\
\hline Não & 89 & 52,0 \\
\hline Ignorada ${ }^{b}$ & 1 & 0,6 \\
\hline \multicolumn{3}{|l|}{ Análise de útero e anexos na necropsiae } \\
\hline Sim & 56 & 69,2 \\
\hline Não & 4 & 4,9 \\
\hline Ignorada ${ }^{b}$ & 21 & 25,9 \\
\hline \multicolumn{3}{|l|}{ Causas do óbito } \\
\hline Causas obstétricas diretas & 79 & 46,2 \\
\hline Hipertensão arterial & 17 & 9,9 \\
\hline Embolia & 12 & 7,0 \\
\hline Hemorragia & 10 & 5,8 \\
\hline Infecção puerperal & 9 & 5,3 \\
\hline Aborto & 8 & 4,7 \\
\hline Gravidez que termina em aborto & 8 & 4,7 \\
\hline Outras complicações relacionadas ao trabalho de parto e parto & 5 & 2,9 \\
\hline Infecção do trato geniturinário na gravidez & 3 & 1,8 \\
\hline Cardiomiopatia no puerpério & 3 & 1,8 \\
\hline Demais causas & 4 & 2,3 \\
\hline
\end{tabular}




\section{Tabela 4 - Frequência das características do puerpério e das circunstâncias do óbito de mulheres que morreram por causas maternas ( $\mathrm{n}=171$ ), Recife, Pernambuco, 2006-2017}

\begin{tabular}{lll}
\hline Características & $\mathbf{n}$ & $\%$ \\
\hline Causas obstétricas indiretas (complicando a gravidez, o parto e o puerpério) & $\mathbf{8 0}$ & $\mathbf{4 6 , 8}$ \\
\hline Doenças do aparelho circulatório & 21 & 12,4 \\
Doenças e afecções especificadas & 18 & 10,5 \\
Doenças do aparelho respiratório & 14 & 8,2 \\
HIV/aids & 7 & 4,1 \\
Hipertensão preexistente & 5 & 2,9 \\
Doenças infecciosas e parasitárias & 4 & 2,3 \\
Transtornos mentais & 4 & 2,3 \\
Doenças do aparelho digestivo & 4 & 2,3 \\
Outras doenças do sangue & 2 & 1,2 \\
Doenças endócrinas & 1 & 0,6 \\
\hline Morte obstétrica de causa não especificada & $\mathbf{3}$ & $\mathbf{1 , 8}$ \\
\hline Morte por qualquer causa obstétrica tardia (43 dias a 1 ano) & $\mathbf{8}$ & $\mathbf{4 , 7}$ \\
\hline Causa mal definida & $\mathbf{1}$ & $\mathbf{0 , 6}$ \\
\hline
\end{tabular}

a) $n=133$ mulheres que morreram no puerpério.

b) Corresponde às alternativas 'Ignorado' $\mathrm{e}^{\prime} \mathrm{Em}$ branco', decorrentes das repostas disponíveis nos instrumentos utilizados.

c) $n=125$ mulheres que sofreram complicações no puerpério.

d) Na ficha de investigação, a variável 'Tipo de complicações do aborto' permite mais de uma resposta.

e) $n=81$ mulheres que passaram por necropsia.

ampliação do nível de escolaridade ou anos de estudo desses óbitos, ${ }^{6-8}$ também identificado aqui. Poucos anos de estudo e ausência de companheiro contribuem para a baixa renda familiar, dificultando a quebra do ciclo de pobreza, 0 acesso à informação e aos serviços de saúde, afora 0 insuficiente suporte emocional da companhia de um parceiro, fatores fundamentais para uma gravidez saudável e o reconhecimento da mulher como sujeito de direito. ${ }^{1,17-19}$

No estudo atual, a maioria das mulheres realizou pré-natal em unidades de Saúde da Família e algumas delas, encaminhadas para o pré-natal de alto risco, não foram atendidas conforme essa indicação. $0 \mathrm{~s} \mathrm{mu-}$ nicípios com elevada cobertura da Atenção Primária à Saúde favorecem a realização do pré-natal nas proximidades da residência, ${ }^{20,21}$ ainda que a visita domiciliar por agentes de saúde e a aferição da pressão arterial não tenham sido observados em todas as mulheres. Falhas como estas, no pré-natal, impedem o diagnóstico precoce e favorecem a ocorrência de complicações na gravidez e no parto. Inquérito nacional sobre parto e nascimento no Brasil identificou que, quando encami- nhadas ao pré-natal de alto risco, $11,5 \%$ das mulheres encontraram dificuldades para esse atendimento. ${ }^{20}$

Verificou-se que algumas mulheres não dispunham do cartão do pré-natal. Embora seja um instrumento de comunicação entre os diferentes níveis de atenção à saúde, fundamental para o acompanhamento clínico do cuidado à saúde materna, os percentuais de mulheres dotadas do Cartão da Gestante mostrou-se abaixo do encontrado em inquérito sobre parto e nascimento no Brasil, segundo o qual $90 \%$ das mulheres afirmaram ter recebido e $72 \%$ apresentaram seu cartão no momento do parto. ${ }^{20}$ Uma assistência integral à saúde pode funcionar como uma política compensatória, capaz de garantir à mulher o acompanhamento adequado da gestação, parto e puerpério., ${ }^{1,19}$

A morte de mulheres com gestação de risco é um marcador de falhas sucessivas, desde um pré-natal inadequado, passando pela busca - nem sempre exitosa - de serviços de maior complexidade para o parto, falta do diagnóstico e da vinculação entre o pré-natal e 0 local do parto. ${ }^{1,4,22,23}$ No presente estudo, observou-se que foram transferidas muitas mulheres, e algumas delas 
passaram por múltiplas transferências no momento do parto/aborto. A ausência da referência no pré-natal para o local do parto faz com que, em muitos casos, a mulher procure atendimento, no momento do parto/aborto, em local sem as devidas condições de atendê-la, fato também identificado em estudo qualitativo desenvolvido no Sudão do Sul. ${ }^{22}$ Essa peregrinação das gestantes foi associada a morbidade materna grave, segundo o inquérito nacional sobre o parto e nascimento de 2012: 19\% das mães que sofreram near miss materno tinham apresentado pelo menos uma complicação clínica ou obstétrica durante a gravidez, no parto ou no período pós-parto. ${ }^{24}$

Quase todos os partos das mulheres falecidas incluídas neste estudo ocorreram em maternidades e foram acompanhados por médicos obstetras, apesar de a maior parte deles sem preenchimento do partograma, além da elevada proporção de partos cesáreos. Revisão internacional sobre o uso do partograma, com 95 pesquisas incluídas, identificou a resistência dos médicos em preenchê-lo, aparentemente indiferentes a sua importância como um instrumento de medida do impacto de seu trabalho; outra conclusão da revisão supracitada foi a necessidade de estabelecer novas estratégias para sua implementação, melhorando, entre outros aspectos, as informações sobre a indicação das cirurgias cesarianas realizadas. ${ }^{25}$ Apesar de o parto e o período puerpério, para a maioria das mulheres, representarem condições fisiológicas a demandar cuidados básicos, podem ocorrer emergências que requeiram respostas rápidas e adequadas, de serviços qualificados, mais complexos, aptos ao enfrentamento do risco de morte materna. ${ }^{1,23} \mathrm{~A}$ transição obstétrica brasileira é caracterizada pela institucionalização dos partos, acompanhando a tendência mundial de medicalização e aumento das intervenções, por vezes desnecessárias. ${ }^{21}$

0 maior número de óbitos encontrado ocorreu no puerpério precoce, período a requerer particular atenção. A vigilância domiciliar quinzenal de puérperas de risco deveria acontecer até dois meses pós-parto, para garantir o diagnóstico, encaminhamento e tratamento oportunos das complicações. ${ }^{26}$

Possivelmente, o menor número de mortes maternas tardias encontrado deva-se às dificuldades de mensuração, em função do preenchimento incorreto das D0s e, por conseguinte, falhas na determinação da causa mortis. ${ }^{23,26}$ Essas dificuldades seriam minimizadas com a ampliação do período de vigilância das puérperas de risco para um ano, associada à investigação dos óbitos de mulheres em idade fértil. ${ }^{27}$ Ademais, a análise do útero e anexos nas necropsias de mulheres em idade fértil contribuiria para 0 conhecimento da real magnitude do problema. No presente estudo, a necropsia foi feita em menos da metade dos casos, e, entre estes, nem todos tiveram o útero e anexos examinados.

As causas obstétricas diretas de óbito analisadas corroboraram pesquisas prévias tendo Recife como cenário. ${ }^{6,13}$ Do total de mortes por aborto, quatro foram referidos como induzidos pela mulher, possivelmente relacionados a práticas inseguras, consequência da condição ilegal do ato no Brasil; o que não impede sua realização, e faz com que muitos desses óbitos não sejam contabilizados como morte materna. ${ }^{29,30}$

As principais causas indiretas de óbito observadas neste estudo foram 'Doenças do aparelho circulatório', 'Doenças do aparelho respiratório', 'Outras doenças e afecções especificadas' e, como quarta causa, 'HIV/aids', todas 'complicando a gravidez, o parto e o puerpério.'. Para a região Nordeste e o Brasil como um todo, sobressaíram 'Outras doenças especificadas'. ${ }^{30}$

Este estudo sugere que não foram assegurados às mulheres os ganhos na saúde materna provenientes dos avanços científicos e tecnológicos. Quanto mais desses avanços forem incorporados no Sistema Único de Saúde (SUS), menor será a incidência das doenças relacionadas à reprodução e mais mortes serão evitadas. ${ }^{1,22,25} 0$ perfil sociodemográfico e assistencial encontrado expressa 0 "consentimento" de mortes maternas por parte da sociedade, ao violar seus direitos básicos relacionados à saúde sexual e reprodutiva, à assistência à saúde de qualidade e oportuna, de acordo com suas necessidades..$^{14,15}$

A maioria das mortes maternas no Recife ocorreu no puerpério, em mulheres negras e sem companheiro. Observou-se elevada proporção de óbitos evitáveis entre as mortes maternas. Destaca-se, dada sua importância para os serviços de saúde materna, a presença de falhas assistenciais no pré-natal, parto e puerpério: dificuldade de acesso à rede, insuficiente acompanhamento, falta de detecção oportuna de complicações, baixa adesão às intervenções baseadas em evidências e às boas práticas, além das questões associadas à vulnerabilidade social $\mathrm{e}$ individual das mulheres. 0s achados sinalizam a urgência em aperfeiçoar a vigilância e atenção à saúde, além do desenvolvimento de estudos com desenhos robustos que avaliem os serviços e explicitem as iniquidades sociais e assistenciais entre diferentes grupos populacionais. 


\section{Contribuição dos autores}

Carvalho PI participou da concepção, delineamento, coleta dos dados, análise e interpretação dos resultados. Frias PG e Vidal SA participaram da concepção, delineamento, análise e interpretação dos resultados. Lemos MLC, Frutuoso LALM, Figueirôa BQ, Pereira CCB e Barreto IC participaram do delineamento, coleta dos dados, análise e interpretação dos resultados. Todos os autores se declaram responsáveis pela redação e revisão crítica do conteúdo intelectual do manuscrito, sua exatidão e integridade, respondendo por ele em quaisquer circunstâncias solicitadas.

\section{Referências}

1. Petersen EE, Davis NL, Goodman D, Cox S, Mayes N, Johnston E, et al. Vital signs: pregnancy-related deaths, United States, 2011-2015, and strategies for prevention, 13 States, 2013-2017. MMWR Morb Mortal Wkly Rep [Internet]. 2019 May [cited 2019 0ct 2];68(18):423-9. Available from: https://www.cdc.gov/mmwr/volumes/68/ $\mathrm{wr} / \mathrm{mm} 6818 \mathrm{e} 1 . \mathrm{htm}$

2. World Health Organization. United Nations Children's Fund. World Bank. United Nations. United Nations Population Division. Trends in maternal mortality: 1990 to 2015: estimates by WHO, UNICEF, UNFPA, World Bank Group and the United Nations Population Division [Internet]. Geneva: World Health Organization; 2015 [cited 2019 Apr 10]. 77 p. Available from: https://apps.who.int/iris/ bitstream/handle/10665/194254/9789241565141_eng. pdf;ssessionid=90B5E6FF8F4F1E08DFD3D62AF9F07B6 2? sequence $=1$

3. Organização das Nações Unidas. Transformando nosso mundo: a agenda 2030 para 0 desenvolvimento sustentável [Internet]. Rio de Janeiro: Nações Unidas; 2015 [citado 2016 fev 18]. 41 p. Disponível em: https://www.undp. org/content/dam/brazil/docs/agenda2030/undp-brAgenda2030-completo-pt-br-2016.pdf

4. Szwarcwald CL, Escalante JJC, Rabello Neto DL, Souza Junior PRB, Victora CG. Estimação da razão de mortalidade materna no Brasil, 2008-2011. Cad Saúde Pública [Internet]. 2014 [citado 2019 out 2];30(Suppl 1):S71-S83. Disponível em: http://www.scielo.br/pdf/csp/v30s1/0102-311Xcsp-30-s1-0071.pdf. doi: 10.1590/0102-311X00125313

5. Zoca B, Escalante JJC, Viola RC, Lecca RCR. Morbimortalidade materna: tendências, causas e investigação de óbitos. In: Ministério da Saúde (BR). Secretaria de Vigilância em
Saúde. Departamento de Análise de Situação em Saúde. Saúde Brasil 2013: uma análise da situação da saúde e das doenças transmissíveis relacionadas à pobreza [Internet]. Brasília: Ministério da Saúde; 2014 [citado 2018 nov 15]. 41-64 p. Disponível em: http://bvsms. saude.gov.br/bvs/publicacoes/saude_brasil_2013_ analise_situacao_saude.pdf

6. Correia RA, Araújo HC, Furtado BMA, Bonfim C. Características epidemiológicas dos óbitos maternos ocorridos em Recife, PE, Brasil (2000-2006). Rev Bras Enferm [Internet]. 2011 jan-fev [citado 2019 out 2];64(1):91-7. Disponível em: http://www.scielo.br/ $\mathrm{pdf} / \mathrm{reben} / \mathrm{v} 64 \mathrm{n} 1 / \mathrm{v} 64 \mathrm{n} 1 \mathrm{a1} 4 . p d f$. doi: 10.1590/S003471672011000100014

7. Martins ACS, Silva LS. Epidemiological profile of maternal mortality. Rev Bras Enferm [Internet]. 2018 [cited 2019 Oct 2];71(Suppl 1):677-83. Available from: http://www. scielo.br/pdf/reben/v71s1/0034-7167-reben-71-s1-0677. pdf. doi: 10.1590/0034-7167-2017-0624

8. Biano RKC, Souza PCB, Ferreira MBG, Silva SR, Ruiz MT. Mortalidade materna no Brasil e nos municípios de Belo Horizonte e Uberaba, 1996 a 2012. Rev Enferm Centro-Oeste Mineiro [Internet]. 2017 [citado 2019 out 2];7:e1464. Disponível em: http://www.seer.ufsj. edu.br/index.php/recom/article/view/1464/1575. doi: 10.19175/recom.v7i0.1464

9. Brasil. Ministério da Saúde. Portaria MS/GM n ${ }^{0} 1.119$, de 5 de junho de 2008. Regulamenta a vigilância de óbitos maternos [Internet]. Diário Oficial União, Brasília (DF), 2008 jun 06 [citado 2019 out 2]; Seção1: 48. Disponível em: http://bvsms.saude.gov.br/bvs/saudelegis/gm/2008/ prt1119_05_06_2008.html 
10. Ministério da Saúde (BR). Secretaria de Vigilância em Saúde. Departamento de Análise de Situação em Saúde. Guia de vigilância epidemiológica do óbito materno [Internet]. Brasília: Ministério da Saúde; 2009 [citado 2019 out 2]. 84 p. Disponível em: http://bvsms.saude.gov.br/bvs/ publicacoes/guia_vigilancia_epidem_obito_materno.pdf

11. Instituto Brasileiro de Geografia e Estatística. Censo demográfico 2010 [Internet]. Rio de Janeiro: Instituto Brasileiro de Geografia e Estatística; 2010 [citado 2018 nov 10]. Disponível em: https://cidades.ibge.gov.br/brasil/ pe/recife/pesquisa/23/25888?detalhes=true $\% 2$ Fhttps: $\% 2$ F\%2Fwww.ibge.gov.br\%2Festatisticas-novoportal\%2Fsocia is\%2Fpopulaca0\%2F9662-censo-demografico-2010.html

12. Ministério da Saúde (BR). Departamento de Informática do SUS. Cadastro nacional de estabelecimentos de saúde: consulta profissional [Internet]. Brasília: Ministério da Saúde; 2019 [citado 2019 abr 1]. Disponível em: http:// cnes.datasus.gov.br/pages/profissionais/consulta.jsp

13. Leite RMB, Araújo TVB, Albuquerque RM, Andrade ARS, Duarte Neto PJ. Fatores de risco para mortalidade materna em área urbana do Nordeste do Brasil. Cad Saúde Publica [Internet]. 2011 out [citado 2019 out 2];27(10):1977-85. Disponivel em: http://www.scielo.br/pdf/csp/v27n10/11. pdf. doi: 10.1590/S0102-311X2011001000011

14. Taquette Stella R. Interseccionalidade de gênero, classe e raça e vulnerabilidade de adolescentes negras às DST/aids. Saúde Soc [Internet]. 2010 [citado 2019 out 2];19(Suppl 2):51-62. Disponível em: http://www. scielo.br/pdf/sausoc/v19s2/06.pdf. doi: 10.1590/S010412902010000600006

15. Pícoli RP, Cazola LHO, Lemos EF. Mortalidade materna segundo raça/cor, em Mato Grosso do Sul, Brasil, de 2010 a 2015. Rev Bras Saúde Matern Infant [Internet]. 2017 out-dez [citado 2019 out 2];17(4):739-47. Disponível em: http://www.scielo.br/pdf/rbsmi/v17n4/ pt_1519-3829-rbsmi-17-04-0729.pdf. doi: 10.1590/180693042017000400007

16. Laurenti R, Mello Jorge MHP, Gotlieb SLD. A mortalidade materna nas capitais brasileiras: algumas características e estimativa de um fator de ajuste. Rev Bras Epidemiol [Internet]. $2004 \mathrm{dez}$ [citado 2019 out 2];7(4):449-60. Disponível em: http://www.scielo.br/pdf/rbepid/v7n4/08. pdf. doi: 10.1590/\$1415-790X2004000400008

17. Hogan MC, Foreman KJ, Naghavi M, Ahn SY, Wang M, Makela SM, et al. Maternal mortality for 181 countries, 1980-2008: a systematic analysis of progress towards millennium development goal 5. Lancet [Internet]. 2010 Apr [cited 2019 Oct 2];375:1609-23. Available from: https://www.thelancet.com/article/S0140-6736(10)605181/abstract. doi: 10.1016/S0140-6736(10)60518-1
18. United Nations. Millennium development goals and beyond 2015 [Internet]. New York: United Nations; 2013 [cited 2016 Dec 7]. Available from: https://www. un.org/millenniumgoals/pdf/Goal_5_fs.pdf

19. Dias JMG, Oliveira APS, Cipolotti R, Monteiro BKSM, Pereira RO. Mortalidade materna. Rev Med Minas Gerais [Internet]. 2015 [citado 2019 out 2];25(2):173-9. Disponível em: http://www.rmmg.org/artigo/detalhes/1771. doi: $10.5935 / 2238-3182.20150034$

20. Viellas EF, Domingues RMSM, Dias MAB, Gama SGN, Theme Filha MM, Costa JV, et al. Assistência pré-natal no Brasil. Cad Saúde Pública [Internet]. 2014 [citado 2019 out 2];30(Supl1):S85-S100. Disponível em: http://www. scielo.br/pdf/csp/v30s1/0102-311X-csp-30-s1-0085.pdf. doi: 10.1590/0102-311X00126013

21. Leal MC, Szwarcwald CL, Almeida PVB, Aquino EML, Barreto ML, Barros F, et al. Saúde reprodutiva, materna, neonatal e infantil nos 30 anos do Sistema Único de Saúde (SUS). Ciênc Saúde Coletiva [Internet]. 2018 jun [citado 2019 out 2];23(6):1915-28. Disponível em: http://www. scielo.br/pdf/csc/v23n6/1413-8123-csc-23-06-1915.pdf. doi: 10.1590/1413-81232018236.03942018

22. Elmusharaf K, Byrne E, AbuAgla A, AbdelRahim A, Manandhar M, Sondorp E, et al. Patterns and determinants of pathways to reach comprehensive emergency obstetric and neonatal care (CEmONC) in South Sudan: qualitative diagrammatic pathway analysis. BMC Pregnancy Childbirth [Internet]. 2017 Aug [cited 2019 0ct 2];17:278. Available from: https://bmcpregnancychildbirth.biomedcentral. com/articles/10.1186/s12884-017-1463-9

23. Vega CEP, Soares VMN, Nasr AMLF. Late maternal mortality: comparison of maternal mortality committees in Brazil. Cad Saúde Publica [Internet]. 2017 [cited 2019 Oct 2];33(3):e00197315. Available from: http://www.scielo. br/pdf/csp/v33n3/1678-4464-csp-33-03-e00197315. pdf. doi: 10.1590/0102-311x00197315

24. Domingues RM, Dias MA, Schilithz AO, Leal MD. Factors associated with maternal near miss in childbirth and the postpartum period: findings from the birth in Brazil National Survey, 2011-2012. Reprod Health [Internet]. 2016 Oct [cited 2019 Oct 2];13(Suppl 3):115. Available from: https://www.ncbi.nlm.nih.gov/pmc/articles/PMC5073804/. doi: 10.1186/s12978-016-0232-y

25. Bedwell C, Levin K, Pett C, Lavender DT. A realist review of the partograph: when and how does it work for labour monitoring? BMC Pregnancy Childbirth [Internet]. 2017 Jan [cited 2019 Oct 2];17:31. Available from: https:// www.ncbi.nlm.nih.gov/pmc/articles/PMC5237234/. doi: 10.1186/s12884-016-1213-4 
26. Ministério da Saúde (BR). Secretaria de Atenção à Saúde. Departamento de Ações Programáticas Estratégicas. Pré-natal e puerpério: atenção qualificada e humanizada [Internet]. Brasília: Ministério da Saúde; 2006 [citado 2019 out 2]. 163 p. (Série A. Normas e Manuais Técnicos); (Série Direitos Sexuais e Direitos Reprodutivos - Caderno no 5). Disponível em: http://bvsms.saude.gov.br/bvs/ publicacoes/manual_pre_natal_puerperio_3ed.pdf

27. Jentoft S, Nielsen VO, Roll-Hansen D. Adjusting maternal mortality data for international comparisons: The case of vital registration systems. Statistics Norway [Internet]. 2014 Mar [cited 2019 Oct 2];773. Available from: https://www.ssb.no/en/forskning/discussion-papers/_ attachment/168855.

28. Victora CG, Aquino EML, Leal MC, Monteiro CA, Barros FC, Szwarcwald CL, et al. Maternal and child health in Brazil: progress and challenges. Lancet [Internet]. 2011 May [cited 2019 0ct 2];377(9780):1863-76. Available

\footnotetext{
Abstract

Objective: to describe the sociodemographic and health care characteristics of women dying due to maternal causes in Recife, Pernambuco, Brazil. Methods: this was a descriptive study using the Mortality Information System, case investigation sheets and summary sheets of early and late maternal deaths occurring between 2006 and 2017, with avoidability assessed by the Municipal Maternal Mortality Committee. Results: we identified 171 deaths, of which 133 were in the puerperium; most deaths occurred among Black women (68.4\%), women without partners (60.2\%), women who had prenatal care (77.2\%), during maternity hospital/general hospital delivery (97.1\%), women attended to by obstetricians (82.6\%);10.4\% of women with puerperal complications bad no health care; avoidable/probably avoidable deaths corresponded to $81.9 \%$, for indirect causes $(n=80)$, and direct causes ( $n=79)$. Conclusion: deaths occurred mainly in the postpartum period, among Black women; care failures were frequent; improved health service surveillance and follow-up is needed in the pregnancypuerperal period, in Recife.

Keywords: Maternal Mortality; Pregnancy Complications; Mortality Registries; Health Information Systems; Epidemiology, Descriptive.
}

from: https://www.thelancet.com/journals/lancet/article/ PIIS0140-6736(11)60138-4/fulltext. doi: 10.1016/ S0140-6736(11)60138-4

29. Martins EF, Almeida PFB, Paixão CO, Bicalho PG, Errico LSP. Causas múltiplas de mortalidade materna relacionada ao aborto no Estado de Minas Gerais, Brasil, 2000-2011. Cad Saúde Pública [Internet]. 2017 [citado 2019 out 2];33(1):e00133115. Disponível em: http://www.scielo. br/pdf/csp/v33n1/1678-4464-csp-33-01-e00133115. pdf. doi: 10.1590/0102-311x00133116

30. Silva BGC, Lima NP, Silva SG, Antúnez SF, Seerig LM, Restrepo-Méndez MC, et al. Mortalidade materna no Brasil no período de 2001 a 2012: tendência temporal e diferenças regionais. Rev Bras Epidemiol [Internet]. 2016 jul-set [citado 2019 out 2];19(3):484-93. Disponível em: http://www.scielo.br/pdf/rbepid/v19n3/19805497-rbepid-19-03-00484.pdf. doi: 10.1590/19805497201600030002

\section{Resumen}

objetivo: describir características sociodemográficas $y$ asistenciales de mujeres que murieron por causa materna en Recife, Pernambuco, Brasil. Métodos: estudio descriptivo utilizando el Sistema de Informaciones sobre Mortalidad, fichas de investigación y síntesis de muertes maternas, tempranas y tardias, entre 2006 y 2017, con evaluación de la evitabilidad por el Comité Municipal de la Mortalidad Materna. Resultados: se identificaron 171 óbitos maternos, 133 en el puerperio; la mayoría de las muertes ocurrió en negras (68,4\%), sin compañero (60,2\%), acompañadas con atención prenatal (77,2\%), de parto en maternidades/hospitales (97,1\%), asistidas por obstetras (82,6\%); de las mujeres con complicaciones puerperales, el 10,4\% no tuvo asistencia; muertes evitables/probablemente evitables correspondieron al $81,9 \%$, por causas indirectas $(n=80)$ y directas $(n=79)$. Conclusión: las muertes ocurrieron principalmente en el período del puerperio y en mujeres negras, con frecuentes fallas en la atención; se requiere una mayor vigilancia y acompañamiento de los servicios de salud en el período de embarazo-puerperio, en Recife.

Palabras clave: Mortalidad Materna; Complicaciones del Embarazo, Registros de Mortalidad; Sistemas de Información en Salud; Epidemiología Descriptiva. 